\title{
Grassmanian and Bosonic Thirring Models with Jump Defects
}

\author{
A.R. Aguirre, J.F. Gomes and A.H. Zimerman ${ }^{1}$ \\ Instituto de Física Teórica - IFT/UNESP \\ Rua Dr. Bento Teobaldo Ferraz, 271, Bloco II \\ CEP 01140-070, São Paulo - SP, Brazil \\ L.H. Ymai ${ }^{2}$ \\ Universidade Federal do Pampa - UNIPAMPA \\ Rua Carlos Barbosa s/n, Bairro Getúlio Vargas, \\ CEP 96412-420, Bagé-RS, Brazil.
}

\begin{abstract}
In this paper we discuss the Lax formulation of the Grassmanian and Bosonic Thirring models in the presence of jump defects. For the Grassmanian case, the defect is described by Bäcklund transformation which is responsible for preserving the integrability of the model. We then propose an extension of the Bäcklund transformation for the Bosonic Thirring model which is verified by some Bäcklund transitions like Vacuum-One soliton, One soliton - One soliton, One soliton - Two solitons and Two solitons - Two solitons. The Lax formulation within the space split by the defect leads to the integrability of Bosonic Thirring model.
\end{abstract}

\section{Introduction}

Recently, there has been some interest in the study of descontinuities (jump defects) in certain integrable field theories. Such discontinuities connects two solutions of two different regions. Bäcklund transformations provide the natural setting for describing integrably such descontinuities since it relates two distinct solutions of the same equation [1]. Examples of such situation can be found for the bosonic $[2,3,4,5,8,6,7]$ and supersymmetric [9], [10] field theories. In a previous note [11] the Grassmanian Thirring model with jump defect was considered by writing its Lagrangian density and the conservation of the modified energy and momentum were established. This last fact has indicated the integrability of the system. In refs. [12], [13] it was shown using the inverse scattering method, that its solutions were not localized with infinite energy. Dispite of this fact, we have reconsidered its study from the point of view of the Lax pair formulation as an instructive guide to discuss more interesting models such as the Bosonic Thirring model.

In section 2 we introduce the Grassmanian Thirring model, as well as its Bäcklund transformation, and discuss the defect contribution for the number of particles. This allows

\footnotetext{
1 aleroagu@ift.unesp.br, jfg@ift.unesp.br, zimerman@ift.unesp.br

${ }^{2}$ lhymai@yahoo.com.br
} 
us to write the Bäcklund transformation in terms of the modified number of particles. We then introduce the Lax pair for each side of the defect and show that it is compatible with Coleman's bosonization formula [14]. We then construct the defect matrix which incorporates the Bäcklund transformation. We determine a gauge transformation connecting both sides of the defect.

In section 3, we give the Lax formulation for the Bosonic Thirring model, present the defect contribution for the number of particles and define the Bäcklund transformation by extending the corresponding expression of the Grassmanian case. The N-soliton solution was presented in [15] by using the inverse scattering method. In subsection 3.1 we present the dressing formulation proposed in [16] in order to obtain in a direct way the Bäcklund solutions. We next show that such formulation naturally determines the defect contribution to the number of particles and therefore the Bäcklund transformation.

In section 4 we verify several examples of the obtained solutions describing Bäcklund transitions: Vacuum-One soliton, One soliton - One soliton, One soliton - Two solitons and Two soliton-Two solitons, obtained by dressing method of section 3.1 .

Finally, in section 5 we write down the modified Lax pair containing the defect and construct a gauge transformation interpolating the two regions.

\section{Grassmanian Thirring Model}

The Lagrangian density for Grassmanian Thirring model can be written as follows,

$$
\begin{aligned}
\mathcal{L}_{p} & =\frac{i}{2} \psi_{1}^{(p)}\left(\partial_{t}-\partial_{x}\right) \psi_{1}^{\dagger(p)}+\frac{i}{2} \psi_{1}^{\dagger(p)}\left(\partial_{t}-\partial_{x}\right) \psi_{1}^{(p)}+\frac{i}{2} \psi_{2}^{(p)}\left(\partial_{t}+\partial_{x}\right) \psi_{2}^{\dagger(p)}+\frac{i}{2} \psi_{2}^{\dagger(p)}\left(\partial_{t}+\partial_{x}\right) \psi_{2}^{(p)} \\
& +m\left(\psi_{1}^{(p)} \psi_{2}^{\dagger(p)}+\psi_{2}^{(p)} \psi_{1}^{\dagger(p)}\right)-g\left(\psi_{1}^{\dagger(p)} \psi_{2}^{\dagger(p)} \psi_{2}^{(p)} \psi_{1}^{(p)}\right) .
\end{aligned}
$$

When considering jump defect at $x=0$ the index $p$ describe the left and right sectors. More explicitly, the lagrangian density for Thirring model describing massive two-component Dirac Grassman fields $\left(\psi_{1}^{(p)}, \psi_{2}^{(p)}\right)$ with $p=1$ corresponding to $x<0, p=2$ corresponding to $x>0$, and $g$ is coupling constant. The field equations for $x \neq 0$ are given by

$$
\begin{aligned}
i\left(\partial_{t}-\partial_{x}\right) \psi_{1}^{(p)} & =m \psi_{2}^{(p)}+g \psi_{2}^{\dagger(p)} \psi_{2}^{(p)} \psi_{1}^{(p)}, \\
i\left(\partial_{t}+\partial_{x}\right) \psi_{2}^{(p)} & =m \psi_{1}^{(p)}+g \psi_{1}^{\dagger(p)} \psi_{1}^{(p)} \psi_{2}^{(p)}, \\
i\left(\partial_{t}-\partial_{x}\right) \psi_{1}^{\dagger(p)} & =-m \psi_{2}^{\dagger(p)}-g \psi_{1}^{\dagger(p)} \psi_{2}^{\dagger(p)} \psi_{2}^{(p)}, \\
i\left(\partial_{t}+\partial_{x}\right) \psi_{2}^{\dagger(p)} & =-m \psi_{1}^{\dagger(p)}-g \psi_{2}^{\dagger(p)} \psi_{1}^{\dagger(p)} \psi_{1}^{(p)},
\end{aligned}
$$

which are the equations of motion for the Grassmanian Thirring model in the bulk. For $x=0$, the equations corresponding to the defect conditions are assumed to be $[11,12]$,

$$
\begin{aligned}
X & =\left(\psi_{1}^{(2)}+\psi_{1}^{(1)}\right)+\frac{i a g}{2 m} \psi_{1}^{(1)} X^{\dagger} X=\left(\psi_{1}^{(2)}+\psi_{1}^{(1)}\right)-\frac{i a g}{2 m} \psi_{1}^{(2)} X^{\dagger} X \\
& =i a^{-1}\left(\psi_{2}^{(2)}-\psi_{2}^{(1)}\right)-\frac{g}{2 m} X^{\dagger} X \psi_{2}^{(2)}=i a^{-1}\left(\psi_{2}^{(2)}-\psi_{2}^{(1)}\right)-\frac{g}{2 m} X^{\dagger} X \psi_{2}^{(1)}
\end{aligned}
$$


its respective hermitian conjugated equations

$$
\begin{aligned}
X^{\dagger} & =\left(\psi_{1}^{\dagger(2)}+\psi_{1}^{\dagger(1)}\right)-\frac{i a g}{2 m} \psi_{1}^{\dagger(1)} X^{\dagger} X=\left(\psi_{1}^{\dagger(2)}+\psi_{1}^{\dagger(1)}\right)+\frac{i a g}{2 m} \psi_{1}^{\dagger(2)} X^{\dagger} X \\
& =-i a^{-1}\left(\psi_{2}^{\dagger(2)}-\psi_{2}^{\dagger(1)}\right)-\frac{g}{2 m} X^{\dagger} X \psi_{2}^{\dagger(2)}=-i a^{-1}\left(\psi_{2}^{\dagger(2)}-\psi_{2}^{\dagger(1)}\right)-\frac{g}{2 m} X^{\dagger} X \psi_{2}^{\dagger(1)},
\end{aligned}
$$

and the time derivatives

$$
\begin{aligned}
\partial_{t} X & =\frac{m}{2 a}\left(\psi_{1}^{(2)}-\psi_{1}^{(1)}\right)-\frac{i m}{2}\left(\psi_{2}^{(2)}+\psi_{2}^{(1)}\right) \\
& -\frac{i g}{4}\left[\psi_{1}^{\dagger(1)} \psi_{1}^{(1)}+\psi_{1}^{\dagger(2)} \psi_{1}^{(2)}+\psi_{2}^{\dagger(1)} \psi_{2}^{(1)}+\psi_{2}^{\dagger(2)} \psi_{2}^{(2)}\right] X \\
\partial_{t} X^{\dagger} & =\frac{m}{2 a}\left(\psi_{1}^{\dagger(2)}-\psi_{1}^{\dagger(1)}\right)+\frac{i m}{2}\left(\psi_{2}^{\dagger(2)}+\psi_{2}^{\dagger(1)}\right) \\
& +\frac{i g}{4}\left[\psi_{1}^{\dagger(1)} \psi_{1}^{(1)}+\psi_{1}^{\dagger(2)} \psi_{1}^{(2)}+\psi_{2}^{\dagger(1)} \psi_{2}^{(1)}+\psi_{2}^{\dagger(2)} \psi_{2}^{(2)}\right] X^{\dagger}
\end{aligned}
$$

These equations correspond precisely to the Bäcklund transformations for the classical Grassmanian Thirring model [12]. The first integral of motion to be considered is the number of particles $N$ given by ,

$$
N=\int_{-\infty}^{0} d x\left(\psi_{1}^{\dagger(1)} \psi_{1}^{(1)}+\psi_{2}^{\dagger(1)} \psi_{2}^{(1)}\right)+\int_{0}^{\infty} d x\left(\psi_{1}^{\dagger(2)} \psi_{1}^{(2)}+\psi_{2}^{\dagger(2)} \psi_{2}^{(2)}\right) .
$$

Using the equations of motion (2.2)-(2.5), we have

$$
\frac{d N}{d t}=\left.\left[\psi_{1}^{\dagger(1)} \psi_{1}^{(1)}-\psi_{2}^{\dagger(1)} \psi_{2}^{(1)}\right]\right|_{x=0}-\left.\left[\psi_{1}^{\dagger(2)} \psi_{1}^{(2)}-\psi_{2}^{\dagger(2)} \psi_{2}^{(2)}\right]\right|_{x=0}
$$

From the Bäcklund transformation and equations of motion we find the modified conserved number of particles,

$$
\mathcal{N}=N+\frac{a}{m} X^{\dagger} X=N+N_{D}, \quad \text { where } \quad N_{D}=\frac{a}{m} X^{\dagger} X
$$

corresponds to the defect contribution to the number of particles. Since $N_{D}^{2}=0,(2.6)$ can be written as

$$
X=\psi_{1}^{(1)} e^{\frac{i g}{4} N_{D}}+\psi_{1}^{(2)} e^{-\frac{i g}{4} N_{D}}=i a^{-1}\left(\psi_{2}^{(2)} e^{\frac{i g}{4} N_{D}}-\psi_{2}^{(1)} e^{-\frac{i g}{4} N_{D}}\right)
$$

The defect contribution to the number of particle quantity $N_{D}$ is the simplest modified conserved charge and turns out to be very important for describing the Bäcklund transformation in an alternative form. The arguments for the integrability of the Grassmaniann Thirring model have already been examined in a previous work [11], by calculating the respective modified conserved energy and momentum quantities. 


\subsection{Lax Pair}

So far we have studied the classical integrability of the Grassmanian Thirring model with jump defect by constructing the lowest conserved quantity, namely, the modified number of particles. The integrability of the model involves also higher conservation laws which are encoded within the Lax pair formalism within the $\hat{s l}(2,1)$ affine Lie algebra and principal gradation (see appendix C), as follows

$$
\mathcal{L}_{t}=i \partial_{t}+A_{t}, \quad \mathcal{L}_{x}=i \partial_{x}+A_{x}
$$

where the connections $A_{t}$ and $A_{x}$ take the following forms

$$
\begin{aligned}
A_{x} & =\frac{g}{2}\left(\psi_{2}^{\dagger} \psi_{2}-\psi_{1}^{\dagger} \psi_{1}\right) h_{1}+\frac{m}{2}\left(\lambda-\lambda^{-1}\right)\left(h_{1}+2 h_{2}\right)-\alpha\left(\psi_{1}^{\dagger} \lambda^{1 / 2}+\psi_{2}^{\dagger} \lambda^{-1 / 2}\right) E_{-\left(\alpha_{1}+\alpha_{2}\right)} \\
& +\alpha\left(\psi_{1} \lambda^{1 / 2}-\psi_{2} \lambda^{-1 / 2}\right) E_{-\alpha_{2}}-\alpha\left(\psi_{1} \lambda^{1 / 2}+\psi_{2} \lambda^{-1 / 2}\right) E_{\alpha_{1}+\alpha_{2}}+\alpha\left(\psi_{1}^{\dagger} \lambda^{1 / 2}-\psi_{2}^{\dagger} \lambda^{-1 / 2}\right) E_{\alpha_{2}} \\
A_{t} & =-\frac{g}{2}\left(\psi_{1}^{\dagger} \psi_{1}+\psi_{2}^{\dagger} \psi_{2}\right) h_{1}+\frac{m}{2}\left(\lambda^{-1}+\lambda\right)\left(h_{1}+2 h_{2}\right)-\alpha\left(\psi_{1}^{\dagger} \lambda^{1 / 2}-\psi_{2}^{\dagger} \lambda^{-1 / 2}\right) E_{-\left(\alpha_{1}+\alpha_{2}\right)} \\
& +\alpha\left(\psi_{1} \lambda^{1 / 2}+\psi_{2} \lambda^{-1 / 2}\right) E_{-\alpha_{2}}-\alpha\left(\psi_{1} \lambda^{1 / 2}-\psi_{2} \lambda^{-1 / 2}\right) E_{\alpha_{1}+\alpha_{2}}+\alpha\left(\psi_{1}^{\dagger} \lambda^{1 / 2}+\psi_{2}^{\dagger} \lambda^{-1 / 2}\right) E_{\alpha_{2}},
\end{aligned}
$$

where we have introduced the parameter $\alpha=\sqrt{\frac{m g}{2}}$. From the zero curvature condition $\left[\mathcal{L}_{t}, \mathcal{L}_{x}\right]=0$, we immediately find the equations of motions (2.2)-(2.5), and the equation corresponding to zero grade in $\lambda$ leads to the following compatibility equation,

$$
i\left(\partial_{t}-\partial_{x}\right)\left(\psi_{1}^{\dagger} \psi_{1}\right)-i\left(\partial_{t}+\partial_{x}\right)\left(\psi_{2}^{\dagger} \psi_{2}\right)+2 m\left(\psi_{2} \psi_{1}^{\dagger}+\psi_{2}^{\dagger} \psi_{1}\right)=0,
$$

which can be rewiten as

$$
i \partial_{t}\left(\psi_{1}^{\dagger} \psi_{1}-\psi_{2}^{\dagger} \psi_{2}\right)-i \partial_{x}\left(\psi_{1}^{\dagger} \psi_{1}+\psi_{2}^{\dagger} \psi_{2}\right)+2 m\left(\psi_{2} \psi_{1}^{\dagger}+\psi_{2}^{\dagger} \psi_{1}\right)=0
$$

Then, remembering that the fermionic currents $j^{\mu}=\bar{\psi} \gamma^{\mu} \psi$ in component fields are given by

$$
j^{0}=\psi_{1}^{\dagger} \psi_{1}+\psi_{2}^{\dagger} \psi_{2}, \quad \text { and } \quad j^{1}=-\psi_{1}^{\dagger} \psi_{1}+\psi_{2}^{\dagger} \psi_{2}
$$

we have

$$
-i \partial_{t} j^{1}-i \partial_{x} j^{0}+2 m\left(\psi_{2}^{\dagger} \psi_{1}-\psi_{1}^{\dagger} \psi_{2}\right)=0
$$

Now, by applying the Coleman's bosonization rules [14]

$$
j^{0}=\frac{\beta}{2 \pi} \partial_{1} \varphi \quad j^{1}=-\frac{\beta}{2 \pi} \partial_{0} \varphi
$$

and using the Mandelstam's operators [17]

$$
\psi_{2}^{\dagger} \psi_{1}=\frac{\hbar}{2 \pi \Lambda}: e^{i \beta \varphi}: \quad \psi_{1}^{\dagger} \psi_{2}=\frac{\hbar}{2 \pi \Lambda}: e^{-i \beta \varphi}:
$$


we obtain

$$
\left(\partial_{t}^{2}-\partial_{x}^{2}\right) \varphi=-\frac{4 m_{0}}{\beta} \sin \beta \varphi
$$

the sine-Gordon equation, where the masses of the two theories are related by

$$
m\left(\frac{\hbar}{\Lambda}\right)=m_{0}
$$

where $\Lambda$ is a cut-off introduced by Mandelstam.

\subsection{Defect Matrix for the Grassmannian Thirring model}

The Thirring Model can be described by the Lax pair (2.16) and (2.17). In the presence of a defect, the integrability of the system is studied by splitting the space into two overlapping regions, $x \leq b$ and $x \geq a$ with $a<b$. Inside the overlap region, $a \leq x \leq b$, we define the Lax pair to be

$$
\begin{aligned}
\hat{A}_{t}^{(1)}= & A_{t}^{(1)}-\theta(x-a)\left[\left\{X-\left(\psi_{1}^{(2)}+\psi_{1}^{(1)}\right)-\frac{i a g}{2 m} X^{\dagger} X \psi_{1}^{(1)}\right\} E_{\left(\alpha_{1}+\alpha_{2}\right)}\right. \\
& \left.+\left\{X^{\dagger}-\left(\psi_{1}^{\dagger(2)}+\psi_{1}^{\dagger(1)}\right)+\frac{i a g}{2 m} X^{\dagger} X \psi_{1}^{\dagger(1)}\right\} E_{-\left(\alpha_{1}+\alpha_{2}\right)}\right] \\
\hat{A}_{x}^{(1)}= & \theta(a-x) A_{x}^{(1)}, \\
\hat{A}_{t}^{(2)}= & A_{t}^{(2)}-\theta(b-x)\left[\left\{X-i a^{-1}\left(\psi_{2}^{(2)}-\psi_{2}^{(1)}\right)+\frac{g}{2 m} X^{\dagger} X \psi_{2}^{(1)}\right\} E_{\alpha_{2}}\right. \\
& \left.+\left\{X^{\dagger}+i a^{-1}\left(\psi_{2}^{\dagger(2)}-\psi_{2}^{\dagger(1)}\right)+\frac{g}{2 m} X^{\dagger} X \psi_{2}^{\dagger(1)}\right\} E_{-\alpha_{2}}\right], \\
\hat{A}_{x}^{(2)}= & \theta(x-b) A_{x}^{(2)} .
\end{aligned}
$$

Within the overlap region, the Lax pair denoted by suffices $p=1,2$, are related by a gauge transformation. Thus, we have

$$
i \partial_{t} K=K \hat{A}_{t}^{(2)}(t, b)-\hat{A}_{t}^{(1)}(t, a) K .
$$

We will assume that the matrix $K$ can be written by the following $\lambda$-expansion,

$$
K=K_{-1 / 2}+K_{0}+K_{1 / 2},
$$

where $K_{i}$ corresponds to an element of grade $\lambda^{i}$. As usual, the entries $K_{i j}$ are calculated grade by grade in a $\lambda$-expansion of the equation (2.30). After some matricial computations, a consistent solution for the defect matrix $K$ is then given in the following form,

$$
K=\left[\begin{array}{ccc}
\lambda^{1 / 2} e^{-\frac{i g a}{2 m} X^{\dagger} X}-\lambda^{-1 / 2}\left(i a^{-1}\right) e^{\frac{i g a}{2 m} X^{\dagger} X} & 0 & \sqrt{\frac{2 g}{m}} X \\
0 & \lambda^{1 / 2} e^{\frac{i g a}{2 m} X^{\dagger} X}-\lambda^{-1 / 2}\left(i a^{-1}\right) e^{\frac{-i g a}{2 m} X^{\dagger} X} & -\sqrt{\frac{2 g}{m}} X^{\dagger} \\
\sqrt{\frac{2 g}{m}} X^{\dagger} & -\sqrt{\frac{2 g}{m}} X & -\lambda^{1 / 2}-\lambda^{-1 / 2}\left(i a^{-1}\right)
\end{array}\right]
$$


where the exponentials can be expanded as

$$
e^{ \pm \frac{i g a}{2 m} X^{\dagger} X}=1 \pm \frac{i g a}{2 m} X^{\dagger} X
$$

and the auxiliary fields $X$ and $X^{\dagger}$ satisfy the equations (2.6)-(2.10) corresponding to the Bäcklund transformations for the Grassmanian Thirring model.

\section{Lax Formulation of the Bosonic Thirring Model}

Now for considering the Bosonic Thirring model, we start from the zero curvature representation using the following Lax pair in the light-cone coordinates ${ }^{3}$,

$$
\mathcal{L}_{\xi}=i \partial_{\xi}+A_{\xi}, \quad \mathcal{L}_{\eta}=i \partial_{\eta}+A_{\eta}
$$

with

$$
\begin{aligned}
& A_{\xi}=\sqrt{m g} \phi_{1} \sigma_{+}^{(0)}-\sqrt{m g} \phi_{1}^{\dagger} \sigma_{-}^{(+1)}-\frac{g}{2}\left(\phi_{1}^{\dagger} \phi_{1}\right) \sigma_{3}^{(0)}-\frac{m}{2} \sigma_{3}^{(+1)}+\alpha_{+} \mathcal{C}, \\
& A_{\eta}=-\sqrt{m g} \phi_{2} \sigma_{+}^{(-1)}+\sqrt{m g} \phi_{2}^{\dagger} \sigma_{-}^{(0)}-\frac{g}{2}\left(\phi_{2}^{\dagger} \phi_{2}\right) \sigma_{3}^{(0)}-\frac{m}{2} \sigma_{3}^{(-1)}+\alpha_{-} \mathcal{C},
\end{aligned}
$$

where $\phi_{i}$ are commuting fields and the Lax connections take values in the $\hat{s} l(2)$-affine KacMoody algebra $\widehat{\mathcal{G}}$ defined by the commutations relations

$$
\begin{aligned}
{\left[\sigma_{3}^{(m)}, \sigma_{3}^{(n)}\right] } & =2 m \mathcal{C} \delta_{m+n, 0}, \\
{\left[\sigma_{3}^{(m)}, \sigma_{ \pm}^{(n)}\right] } & = \pm 2 \sigma_{ \pm}^{(m+n)} \\
{\left[\sigma_{+}^{(m)}, \sigma_{-}^{(n)}\right] } & =\sigma_{3}^{(m+n)}+m \mathcal{C} \delta_{m+n, 0}, \\
{\left[d, T^{(n)}\right] } & =n T^{(n)}, \quad T^{(n)} \equiv \sigma_{3}^{(m)}, \sigma_{ \pm}^{(n)} .
\end{aligned}
$$

where $\mathcal{C}$ represents the central term. The principal grading for the $\hat{s} l(2)$ is generated by the operator

$$
Q=2 d+\frac{1}{2} \sigma_{3}^{(0)}
$$

The grading operator $Q$ decomposes the algebra $\widehat{\mathcal{G}}$ into subalgebras generated by elements of positive, negative and zero grades respectively,

$$
\widehat{\mathcal{G}}=\widehat{\mathcal{G}}_{+} \oplus \widehat{\mathcal{G}}_{0} \oplus \widehat{\mathcal{G}}_{-} .
$$

From the zero curvature condition $\left[\mathcal{L}_{\xi}, \mathcal{L}_{\eta}\right]=0$, we get the field equations for the Bosonic Thirring model

$$
\begin{aligned}
i \partial_{\eta} \phi_{1} & =m \phi_{2}+g\left(\phi_{2}^{\dagger} \phi_{2}\right) \phi_{1}, \\
i \partial_{\xi} \phi_{2} & =m \phi_{1}+g\left(\phi_{1}^{\dagger} \phi_{1}\right) \phi_{2}, \\
i \partial_{\eta} \phi_{1}^{\dagger} & =-m \phi_{2}^{\dagger}-g\left(\phi_{2}^{\dagger} \phi_{2}\right) \phi_{1}^{\dagger}, \\
i \partial_{\xi} \phi_{2}^{\dagger} & =-m \phi_{1}^{\dagger}-g\left(\phi_{1}^{\dagger} \phi_{1}\right) \phi_{2}^{\dagger},
\end{aligned}
$$

\footnotetext{
${ }^{3}$ Here, we use the light-cone coordinates $\xi=\frac{1}{2}(t+x), \eta=\frac{1}{2}(t-x)$.
} 
together with the following equations

$$
\begin{aligned}
i \partial_{\xi}\left(\phi_{2}^{\dagger} \phi_{2}\right)-i \partial_{\eta}\left(\phi_{1}^{\dagger} \phi_{1}\right)-2 m\left(\phi_{1}^{\dagger} \phi_{2}-\phi_{2}^{\dagger} \phi_{1}\right) & =0, \\
i \partial_{\xi} \alpha_{-}-i \partial_{\eta} \alpha_{+}+m g\left(\phi_{1}^{\dagger} \phi_{2}\right)+\frac{m^{2}}{2} & =0 .
\end{aligned}
$$

Equation (3.10) is a straightforward consequence of the field equations (3.6)-(3.9), and the Eq.(3.11) determines the dependence of the fields $\alpha_{ \pm}$in terms of the massive fields $\phi$ 's. The conserved quantity which gives the number of particles in the bulk is given by

$$
N=\int_{-\infty}^{\infty} d x\left[\phi_{1}^{\dagger} \phi_{1}+\phi_{2}^{\dagger} \phi_{2}\right]
$$

Let $N_{D}$ be the defect contribution to the number of particles density, which satisfies

$$
\frac{d N_{D}}{d t}=-\left.\left[\phi_{1}^{\dagger(1)} \phi_{1}^{(1)}-\phi_{2}^{\dagger(1)} \phi_{2}^{(1)}\right]\right|_{x=0}+\left.\left[\phi_{1}^{\dagger(2)} \phi_{1}^{(2)}-\phi_{2}^{\dagger(2)} \phi_{2}^{(2)}\right]\right|_{x=0}
$$

The auxiliary functions $X$ defining the Bäcklund transformation will be assumed, according to (2.14), to satisfy the following algebraic relations,

$$
X=\phi_{1}^{(1)} \exp \left[\frac{i g N_{D}}{4}\right]+\phi_{1}^{(2)} \exp \left[\frac{-i g N_{D}}{4}\right]=\frac{i}{a}\left[\phi_{2}^{(2)} \exp \left[\frac{i g N_{D}}{4}\right]-\phi_{2}^{(1)} \exp \left[\frac{-i g N_{D}}{4}\right]\right],
$$

and

$$
X^{\dagger}=\phi_{1}^{\dagger(1)} \exp \left[-\frac{i g N_{D}}{4}\right]+\phi_{1}^{\dagger(2)} \exp \left[\frac{i g N_{D}}{4}\right]=\frac{-i}{a}\left[\phi_{2}^{\dagger(2)} \exp \left[\frac{-i g N_{D}}{4}\right]-\phi_{2}^{\dagger(1)} \exp \left[\frac{i g N_{D}}{4}\right]\right]
$$

As a consequence of (3.6)-(3.9) in (3.15), we also can obtain the following relations,

$$
\begin{aligned}
& \partial_{\xi} X=m a^{-1}\left(\phi_{1}^{(2)} \exp \left[\frac{i g N_{D}}{4}\right]-\phi_{1}^{(1)} \exp \left[\frac{-i g N_{D}}{4}\right]\right)-\frac{i g}{2}\left(\phi_{1}^{\dagger(1)} \phi_{1}^{(1)}+\phi_{1}^{\dagger(2)} \phi_{1}^{(2)}\right) X \\
& \partial_{\eta} X=-i m\left(\phi_{2}^{(1)} \exp \left[\frac{i g N_{D}}{4}\right]+\phi_{2}^{(2)} \exp \left[\frac{-i g N_{D}}{4}\right]\right)-\frac{i g}{2}\left(\phi_{2}^{\dagger(1)} \phi_{2}^{(1)}+\phi_{2}^{\dagger(2)} \phi_{2}^{(2)}\right) X .
\end{aligned}
$$

The integrability condition for the above equations,

$$
\partial_{\xi}\left(\partial_{\eta} X\right)=\partial_{\eta}\left(\partial_{\xi} X\right)
$$

implies that both $\phi_{i}^{(1)}$ as well as $\phi_{i}^{(2)}$ are solutions of the equations of motion (3.6)-(3.9) for the Bosonic Thirring model. Then, we identify the eqs. (3.15)-(3.17) to be the auto-Bäcklund transformations for the Bosonic Thirring model. These transformations have shown to be totally consistent. Using Eqs. (3.14)-(3.17) we found that the defect contribution for the number of particles in this case can be written as,

$$
N_{D}=\frac{2}{g} \arcsin \left[\frac{g a}{2 m} X^{\dagger} X\right] .
$$

Notice that taking a naive Grassmaniann limit, $N_{D}$ given by Eq. (3.19) is consistent with the expression given in Eq. (2.13). 


\subsection{Dresssing Formulation}

We now present a systematic way of constructing solutions by the dressing procedure of refs. [16]. Its advantage is to provide a direct connection between certain quantities naturally appearing within the dressing formalism and in the Bäcklund transformation as we shall see in (4.1) and (4.2). The key ingredient is the existence of two gauge transformations $\Theta_{+}=\exp \left(\mathcal{G}_{\geq}\right)$and $\Theta_{-}=\exp \left(\mathcal{G}_{\leq}\right)$mapping the vacuum in a non-trivial configuration, i.e.,

$$
A_{\mu}^{v a c} \longrightarrow A_{\mu} \equiv \Theta_{ \pm}^{-1} i \partial_{\mu} \Theta_{ \pm}+\Theta_{ \pm}^{-1} A_{\mu}^{v a c} \Theta_{ \pm}, \quad \mu=\{\eta, \xi\}
$$

As consequence of the graded structure, the form of the Lax connection is preserved by these transformations. Since $A_{\mu}$ and $A_{\mu}^{v a c}$ satisfy the zero curvature condition, they are of the form

$$
A_{\mu}=i T \partial_{\mu} T^{-1}, \quad A_{\mu}^{v a c}=i T_{0} \partial_{\mu} T_{0}^{-1}
$$

where $T$ and $T_{0}$ are group elements. From the equivalence of the two dressing transformations (3.20) we find that

$$
\Theta_{-} \Theta_{+}^{-1}=T_{0} \rho T_{0}^{-1}
$$

where $\rho$ is a constant group element. In order to construct sistematically soliton solutions we now define the vacuum configuration,

$$
\phi_{1}^{(0)}=\phi_{2}^{(0)}=\phi_{1}^{\dagger(0)}=\phi_{2}^{\dagger(0)}=0, \quad \alpha_{+}^{(0)}=\frac{-i m^{2} \eta}{4}, \quad \alpha_{-}^{(0)}=\frac{i m^{2} \xi}{4} .
$$

and Lax connections (3.2) become

$$
A_{\xi}^{v a c}=-\frac{m}{2} \sigma_{3}^{(+1)}+\frac{-i m^{2} \eta}{4} \mathcal{C}, \quad A_{\eta}^{v a c}=-\frac{m}{2} \sigma_{3}^{(-1)}+\frac{i m^{2} \xi}{4} \mathcal{C} .
$$

They are associated to the following linear problem

$$
i \partial_{\eta} T_{0}=-A_{\eta}^{v a c} T_{0}, \quad i \partial_{\xi} T_{0}=-A_{\xi}^{v a c} T_{0},
$$

which is solved as follows

$$
T_{0}=e^{-i \eta E^{-}-i \xi E^{+}}, \quad \text { with } \quad E^{ \pm} \equiv \frac{m}{2} \sigma_{3}^{( \pm 1)} .
$$

The dressing matrices $\Theta_{ \pm}$are now determined by the gauge transformation (3.20) with

$$
\Theta_{+}=e^{m(0)} e^{m(1)} e^{m(2)} \ldots \quad \Theta_{-}=e^{l(0)} e^{l(-1)} e^{l(-2)} \ldots
$$

where $\Theta_{+}$is constructed from elements $m(k)$ of a subalgebra containing grade $k \geq 0$, while $\Theta_{-}$is constructed from elements $l(k)$ of a subalgebra containing grade $k \leq 0$. From eq.(3.20) we get the following results for the first few elements $m(k)$ and $l(k)$

$$
\begin{aligned}
m(0) & =\chi_{+} \sigma_{3}^{(0)}+\nu_{+} \mathcal{C}, \quad l(0)=\left(i \pi-\chi_{+}\right) \sigma_{3}^{(0)}+\nu_{-} \mathcal{C}, \\
m(1) & =\sqrt{\frac{g}{m}}\left[\phi_{2} \sigma_{+}^{(0)}+\phi_{2}^{\dagger} \sigma_{-}^{(1)}\right], \quad l(-1)=-\sqrt{\frac{g}{m}}\left[\phi_{1}^{\dagger} \sigma_{-}^{(0)}+\phi_{1} \sigma_{+}^{(-1)}\right] \\
m(2) & =a_{+} \sigma_{3}^{(1)}, \quad l(-2)=a_{-} \sigma_{3}^{(-1)},
\end{aligned}
$$


where the fields $\phi$ 's satisfy the equations of motion(3.6)-(3.9), and the fields $\chi_{+}, \nu_{ \pm}, a_{ \pm}$satisfy the following equations,

$$
\begin{array}{rlrl}
i \partial_{\xi} \chi_{+} & =-\frac{g}{2}\left(\phi_{1}^{\dagger} \phi_{1}\right), & i \partial_{\eta} \chi_{+}=\frac{g}{2}\left(\phi_{2}^{\dagger} \phi_{2}\right), \\
i \partial_{\xi} \nu_{+}=\alpha_{+}-\alpha_{+}^{(0)}, & i \partial_{\eta} \nu_{+}=\alpha_{-}-\alpha_{-}^{(0)}-m a_{+}-\frac{g}{2}\left(\phi_{2}^{\dagger} \phi_{2}\right), \\
i \partial_{\eta} \nu_{-}=\alpha_{-}-\alpha_{-}^{(0)}, & i \partial_{\xi} \nu_{-}=\alpha_{+}-\alpha_{+}^{(0)}+m a_{-}-\frac{g}{2}\left(\phi_{1}^{\dagger} \phi_{1}\right) .
\end{array}
$$

The $(\xi, \eta)$-dependence of the fields is given explicitly by the right-hand-side of Eq.(3.22). In fact, the solutions can be calculated by taking the expectation value between states of a given representation of $\widehat{\mathcal{G}}$. As usual, we consider the highest weight representation of the $\hat{s} l(2)$. Firstly, let $\left|\lambda_{0}\right\rangle$ and $\left|\lambda_{1}\right\rangle$ be the corresponding highest weight states of $\hat{s} l(2)$, and define the $\tau$-functions as follows

$$
\tau_{i}=\left\langle\lambda_{i}\left|\Theta_{-} \Theta_{+}^{-1}\right| \lambda_{i}\right\rangle=\left\langle\lambda_{i}\left|T_{0} \rho T_{0}^{-1}\right| \lambda_{i}\right\rangle, \quad i=1,2
$$

The soliton solutions are obtained by choosing the constant element $\rho=e^{V}$, as the exponential of an eigenvector $V$ of the elements of algebra $E^{ \pm}$. This eigenvector can be constructed in the following way

$$
V_{ \pm}(\gamma)=\sum_{n \in \mathbb{Z}} \gamma^{-n} \sigma_{ \pm}^{(n)}
$$

satisfying the following commutation relations

$$
\begin{aligned}
& {\left[E^{+}, V_{ \pm}(\gamma)\right]= \pm m \gamma V_{ \pm}(\gamma)} \\
& {\left[E^{-}, V_{ \pm}(\gamma)\right]= \pm \frac{m}{\gamma} V_{ \pm}(\gamma)}
\end{aligned}
$$

Its clear from (3.36)-(3.37) that $V_{+}(\gamma)$ and $V_{-}(-\gamma)$ have the same eigenvalue. From (3.22) we obtain

$$
T_{0} e^{\mu_{ \pm} V_{ \pm}(\gamma)} T_{0}^{-1}=\exp \left[e^{\mp \Gamma} \mu_{ \pm} V_{ \pm}(\gamma)\right] \cong 1+\mu_{ \pm} e^{\mp \Gamma} V_{ \pm}(\gamma)
$$

with $\Gamma=i m\left(\xi \gamma+\gamma^{-1} \eta\right)$. This construction corresponds to the Fubini-Veneziano vertex operator which satisfies

$$
V_{+}\left(\gamma_{1}\right) V_{+}\left(\gamma_{2}\right) \rightarrow 0, \quad V_{-}\left(\gamma_{1}\right) V_{-}\left(\gamma_{2}\right) \rightarrow 0, \quad \text { as } \quad \gamma_{1} \rightarrow \gamma_{2}
$$

In general, the N-soliton solution is obtained taking $\rho=e^{\mu_{1} V\left(\gamma_{1}\right)} e^{\mu_{2} V\left(\gamma_{2}\right)} \cdots e^{\mu_{N} V\left(\gamma_{N}\right)}$, being $\mu_{k}$ some arbitrary parameters and the vertex functions satisfy the following commutation relation

$$
\left[E^{(n)}, V\left(\gamma_{k}\right)\right]=f\left(n, \gamma_{k}\right) V\left(\gamma_{k}\right)
$$




\section{Bäcklund Solutions}

In this section, we want to discuss the type of solutions derived from the auto-Bäcklund transformations (3.15)-(3.17). Particularly, we are interested to show that these solutions are in totally consistency with the ones given by the dressing method. Firstly, we noted that there is a closed relation between the field $\chi_{+}$appearing in the dressing procedure and the defect contribution to the number of particle conserved quantity $N_{D}$. In fact, from eqs. (3.31) it follows that

$$
\frac{\partial \chi_{+}}{\partial t}=\frac{i g}{4}\left(\phi_{1}^{\dagger} \phi_{1}-\phi_{2}^{\dagger} \phi_{2}\right)
$$

Comparing with eqn. (3.13) we can take,

$$
N_{D}=\left.\frac{4 i}{g}\left(\chi_{+}^{(1)}-\chi_{+}^{(2)}\right)\right|_{x=0}
$$

where $\chi_{+}^{(i)}$ is given in general by eqn. (A.1). So, this relation gives us a connection between the dressing solutions and the Bäcklund solutions.

\subsection{Vacuum - one-soliton solution}

In order to investigate this connection, we will consider the situation of performing the Bäcklund transformation starting from the vacuum solution,

$$
\phi_{1}^{(1)}=\phi_{2}^{(1)}=\phi_{1}^{\dagger(1)}=\phi_{2}^{\dagger(1)}=0, \quad \chi_{+}^{(1)}=\frac{i \pi}{2} .
$$

From the explicit form of $\chi_{+}^{(2)}$ for one-soliton solution given by (A.8) and using the Bäcklund transformations (3.15)-(3.17), we obtained

$$
X=\sqrt{\frac{m}{g}}\left[\frac{-i \mu_{1}^{(2)} a^{-1} e^{-\Gamma_{2}}}{\left[1+\left|\Omega_{2}\right|^{2} e^{-4 \Gamma_{2}}\right]^{\frac{1}{2}}}\right], \quad X^{\dagger}=\sqrt{\frac{m}{g}}\left[\frac{i \mu_{1}^{*(2)} a^{-1} e^{-\Gamma_{2}}}{\left[1+\left|\Omega_{2}\right|^{2} e^{-4 \Gamma_{2}}\right]^{\frac{1}{2}}}\right],
$$

and

$$
\begin{array}{rlrl}
\phi_{1}^{(2)} & =\sqrt{\frac{m}{g}}\left[\frac{i \mu_{1}^{(2)} a^{-1} e^{-\Gamma_{2}}}{1+\Omega_{2} e^{-2 \Gamma_{2}}}\right], & \phi_{2}^{(2)}=\sqrt{\frac{m}{g}}\left[\frac{\mu_{1}^{(2)} e^{-\Gamma_{2}}}{1-\Omega_{2} e^{-2 \Gamma_{2}}}\right], \\
\phi_{1}^{\dagger(2)}=-\sqrt{\frac{m}{g}}\left[\frac{i \mu_{1}^{*(2)} a^{-1} e^{-\Gamma_{2}}}{1-\Omega_{2} e^{-2 \Gamma_{2}}}\right], & \phi_{2}^{\dagger(2)}=\sqrt{\frac{m}{g}}\left[\frac{\mu_{1}^{*(2)} e^{-\Gamma_{2}}}{1+\Omega_{2} e^{-2 \Gamma_{2}}}\right] .
\end{array}
$$

where $\mu_{1}^{(2)}$ is an arbitrary constant. Thus, we have found exactly the one-soliton solution for the BTM firstly obtained by the Dressing method, with $\gamma_{2}=i a^{-1}$. Then, it shows that our Bäcklund transformation are compatible not only with the integrability of the Bosonic Thirring model in the presence of a jump-defect, but with the soliton solutions obtained by dressing method, too. 


\subsection{One-soliton - one-soliton Solution}

We are now interested in investigating the situation of one soliton approaches a defect and on the other side of it we have an outgoing soliton. Then, we consider the form of the one-soliton solution given by (A.9),

$$
\phi_{1}^{(k)}=\sqrt{\frac{m}{g}}\left[\frac{\mu_{1}^{(k)} \gamma_{k} e^{-\Gamma_{k}}}{1+\Omega_{k} e^{-2 \Gamma_{k}}}\right], \quad \phi_{2}^{(k)}=\sqrt{\frac{m}{g}}\left[\frac{\mu_{1}^{(k)} e^{-\Gamma_{k}}}{1-\Omega_{k} e^{-2 \Gamma_{k}}}\right], \quad k=1,2,
$$

where $\mu_{1}^{(k)}$ is a parameter corresponding to each side around the defect. From (3.15) we find

$$
\begin{array}{r}
\mu_{1}^{(1)} \gamma_{1} e^{-\Gamma_{1}}+\mu_{1}^{(2)} \gamma_{2} e^{-\Gamma_{2}}-\mu_{1}^{(1)} \gamma_{1} \Omega_{2} e^{-\Gamma_{1}-2 \Gamma_{2}}-\mu_{1}^{(2)} \gamma_{2} \Omega_{1} e^{-\Gamma_{2}-2 \Gamma_{1}}= \\
-i a^{-1} \mu_{1}^{(1)} e^{-\Gamma_{1}}+i a^{-1} \mu_{1}^{(2)} e^{-\Gamma_{2}}-i a^{-1} \mu_{1}^{(1)} \Omega_{2} e^{-\Gamma_{1}-2 \Gamma_{2}}+i a^{-1} \mu_{1}^{(2)} \Omega_{1} e^{-\Gamma_{2}-2 \Gamma_{1}},
\end{array}
$$

with $a$ being the Bäcklund parameter. This relation implies the following conditions over the parameters,

$$
\gamma_{1}=\gamma_{2}=\gamma, \quad \mu_{1}^{(1)}=\left[\frac{\sigma-\gamma}{\sigma+\gamma}\right] \mu_{1}^{(2)}, \quad \Omega_{1}=\left[\frac{\sigma-\gamma}{\sigma+\gamma}\right]^{2} \Omega_{2},
$$

where we have defined $\sigma=i a^{-1}$. Then, an important point that can be noted is that the jump-defect preserves the soliton velocity and the only effect of the interaction soliton-defect is a phase shift. In addition, we also have that the limiting cases when $\mu_{1}^{(1)}=0$ or $\mu_{1}^{(2)}=0$ do exist, and correspond to the situation where $\gamma=|\sigma|$ with $a>0$, and $\gamma=|\sigma|$ with $a<0$ respectively. Clearly, these cases indicate creation and absorption of the soliton. As $a \rightarrow \infty$, the parameter $\mu_{1}^{(1)} \rightarrow-\mu_{1}^{(2)}$, which means that if the defect parameter is large the soliton

will invert its shape. As $a \rightarrow 0$, we obtain $\mu_{1}^{(1)}=\mu_{1}^{(2)}$, indicating that there is not defect and the soliton shape is preserved as expected. Some of these features have already been found for several integrable models with defects $[5,9]$.

\subsection{One-soliton - two-soliton Solution}

In this case we want to examine the situation when one-soliton is incoming and two-solitons are outgoing. From Eqs.(A.7) and (A.10), we can write the one-soliton solution with parameter $\gamma_{1}$ in terms of the tau-functions as follows,

$$
\begin{aligned}
& \tau_{0}^{(1)}=1-\frac{\mu_{1}^{(1)} \mu_{2}^{(1)}}{4} e^{-2 \Gamma_{1}}, \quad \tau_{1}^{(1)}=1+\frac{\mu_{1}^{(1)} \mu_{2}^{(1)}}{4} e^{-2 \Gamma_{1}}, \quad \tau_{2}^{(1)}=\mu_{1}^{(1)} \gamma_{1} e^{-\Gamma_{1}}, \\
& \tau_{3}^{(1)}=\mu_{2}^{(1)} e^{-\Gamma_{1}}, \quad \tau_{4}=\mu_{1}^{(1)} e^{-\Gamma_{1}}, \quad \tau_{5}^{(1)}=\frac{\mu_{2}^{(1)}}{\gamma_{1}} e^{-\Gamma_{1}} .
\end{aligned}
$$

In addition, we consider the two-soliton solution (B.22)-(B.27), with parameters $\gamma_{1}=-\gamma_{2}$ and $\gamma_{3}=-\gamma_{4}$. From the Bäcklund relation (3.14) we obtain the following equation in terms of the tau-functions,

$$
\left[\tau_{2}^{(1)} \tau_{0}^{(2)}+\tau_{2}^{(2)} \tau_{0}^{(1)}\right]-\sigma\left[\tau_{4}^{(2)} \tau_{1}^{(1)}-\tau_{4}^{(1)} \tau_{1}^{(2)}\right]=0
$$


and from Eq. (3.15) we get,

$$
\left[\tau_{3}^{(1)} \tau_{1}^{(2)}+\tau_{3}^{(2)} \tau_{1}^{(1)}\right]+\sigma\left[\tau_{5}^{(2)} \tau_{0}^{(1)}-\tau_{5}^{(1)} \tau_{0}^{(2)}\right]=0,
$$

where $\sigma=i a^{-1}$ is the parameter associated to the Bäcklund transformation. It is worth mentioning that the Eqs. (4.12) and (4.13) hold for all Bäcklund transitions and not only for the present case, which are a direct consequence of the expressions (A.1)-(A.4). After some

calculations, we found that the equation (4.12) is satisfied provided the following relations hold,

$$
\sigma=\gamma_{3}, \quad \mu_{1}^{(2)}=\left[\frac{\gamma_{3}+\gamma_{1}}{\gamma_{3}-\gamma_{1}}\right] \mu_{1}^{(1)}, \quad \mu_{2}^{(2)}=\left[\frac{\gamma_{3}+\gamma_{1}}{\gamma_{3}-\gamma_{1}}\right] \mu_{2}^{(1)} .
$$

Hence, the parameter of the Bäcklund transformation must to be exactly the second parameter $\gamma_{3}$ of the two-soliton solution. Moreover, note that there are no conditions imposed over the parameters $\mu_{3}^{(2)}$ and $\mu_{4}^{(2)}$ because of our choice of the parameter $\gamma_{1}$ as the parameter for the one-soliton solution. In fact, if we choose $\gamma_{3}$ as the parameter for the one-soliton solution in (4.10)-(4.11) we get the following conditions from the Eq.(4.12),

$$
\sigma=\gamma_{1}, \quad \mu_{3}^{(2)}=\left[\frac{\gamma_{3}+\gamma_{1}}{\gamma_{3}-\gamma_{1}}\right] \mu_{1}^{(1)}, \quad \mu_{4}^{(2)}=\left[\frac{\gamma_{3}+\gamma_{1}}{\gamma_{3}-\gamma_{1}}\right] \mu_{2}^{(1)}
$$

In this case, the parameter of the Bäcklund transformation must to be $\gamma_{1}$ and $\mu_{1}^{(2)}$ and $\mu_{2}^{(2)}$ are arbitraries. These results can be put together on a compact form by taking $\gamma$ as the one-soliton solution parameter and $\sigma$ the Bäcklund parameter,

$$
\mu_{1}^{(2)}=-\mu_{3}^{(2)}=\left[\frac{\sigma+\gamma}{\sigma-\gamma}\right] \mu_{1}^{(1)}, \quad \mu_{2}^{(2)}=-\mu_{4}^{(2)}=\left[\frac{\sigma+\gamma}{\sigma-\gamma}\right] \mu_{2}^{(1)},
$$

where $\gamma$ and $\sigma$ can take the values $\left\{\gamma_{1}, \gamma_{3}\right\}$. In others words, these results is providing us of an indirectly evidence of the permutability theorem of the Bäcklund transformations.

\subsection{Two soliton-two soliton Solution}

For this time we consider the two soliton solution given explicitely by the Eqs. (B.22)-(B.27) for both sides of the defect. Then, the two Bäcklund relations (4.12) and (4.13) are satisfied provided the following realtions between the parameters hold,

$$
\begin{aligned}
\mu_{1}^{(1)} & =\left[\frac{\sigma-\gamma_{1}}{\sigma+\gamma_{1}}\right] \mu_{1}^{(2)}, & \mu_{3}^{(1)} & =\left[\frac{\sigma-\gamma_{3}}{\sigma+\gamma_{3}}\right] \mu_{3}^{(2)}, \\
\mu_{2}^{(1)} & =\left[\frac{\sigma-\gamma_{1}}{\sigma+\gamma_{1}}\right] \mu_{2}^{(2)}, & \mu_{4}^{(1)} & =\left[\frac{\sigma-\gamma_{3}}{\sigma+\gamma_{3}}\right] \mu_{4}^{(2)}
\end{aligned}
$$

defining the corresponding phase shifts. 


\section{$5 \quad$ Defect Matrix for the Bosonic Thirring Model}

In order to calculate the defect matrix, it is necessary to introduce the modified Lax pair defined in two overlapping regions containing the defect. Following $[2,4]$, the space can be splitted by introducing two points $0<a<b$ which define two regions : on the left $R^{(1)}$, $-\infty<x<b$, and on the right $R^{(2)}, a<x<\infty$. Then, the modified Lax pair can be written in a general form, as follows

$$
\begin{aligned}
& \hat{A}_{t}^{(1)}=A_{t}^{(1)}+\theta(x-a)\left[F_{1}\left(\phi_{1}^{(1)}, \phi_{1}^{\dagger(2)}, X\right) \sigma_{+}^{(0)}-F_{1}^{\dagger}\left(\phi_{1}^{\dagger(1)}, \phi_{1}^{\dagger(2)}, X^{\dagger}\right) \sigma_{-}^{(+1)}\right], \\
& \hat{A}_{x}^{(1)}=A_{x}^{(1)} \theta(a-x), \\
& \hat{A}_{t}^{(2)}=A_{t}^{(2)}+\theta(b-x)\left[F_{2}\left(\phi_{2}^{(1)}, \phi_{2}^{(2)}, X\right) \sigma_{+}^{(-1)}-F_{2}^{\dagger}\left(\phi_{2}^{\dagger(1)}, \phi_{2}^{\dagger(2)}, X^{\dagger}\right) \sigma_{-}^{(0)}\right], \\
& \hat{A}_{x}^{(2)}=A_{x}^{(2)} \theta(x-b) .
\end{aligned}
$$

where $A_{t}$ and $A_{x}$ are related with the connections introduced in (3.2) by the following simple relations,

$$
A_{t}^{(p)}=\frac{1}{2}\left(A_{\xi}^{(p)}+A_{\eta}^{(p)}\right), \quad A_{x}^{(p)}=\frac{1}{2}\left(A_{\xi}^{(p)}-A_{\eta}^{(p)}\right), \quad p=1,2,
$$

and the functions $F_{i}$ and $F_{i}^{\dagger}$ are given explicitly by

$$
\begin{aligned}
F_{1}\left(\phi_{1}^{(1)}, \phi_{1}^{\dagger(2)}, X\right) & =X-\phi_{1}^{(1)} \exp \left[\frac{i g N_{D}}{4}\right]-\phi_{1}^{(2)} \exp \left[\frac{-i g N_{D}}{4}\right], \\
F_{1}^{\dagger}\left(\phi_{1}^{\dagger(1)}, \phi_{1}^{\dagger(2)}, X^{\dagger}\right) & =X^{\dagger}-\phi_{1}^{\dagger(1)} \exp \left[\frac{-i g N_{D}}{4}\right]-\phi_{1}^{\dagger(2)} \exp \left[\frac{i g N_{D}}{4}\right], \\
F_{2}\left(\phi_{2}^{(1)}, \phi_{2}^{(2)}, X\right) & =X-i a^{-1}\left[\phi_{2}^{(2)} \exp \left[\frac{i g N_{D}}{4}\right]-\phi_{2}^{(1)} \exp \left[\frac{-i g N_{D}}{4}\right]\right], \\
F_{2}^{\dagger}\left(\phi_{2}^{\dagger(1)}, \phi_{2}^{\dagger(2)}, X^{\dagger}\right) & =X^{\dagger}+i a^{-1}\left[\phi_{2}^{\dagger(2)} \exp \left[\frac{-i g N_{D}}{4}\right]-\phi_{2}^{\dagger(1)} \exp \left[\frac{i g N_{D}}{4}\right]\right] .
\end{aligned}
$$

These modified Lax pair allow us to derive the equations of motion for each region after applying the zero curvature condition, and the functions $F_{i}$ and $F_{i}^{\dagger}$ provide us the defect relations or auto-Bäcklund transformations in the same way. So, within the overlap region the modified Lax pair are related by the gauge transformation,

$$
i \partial_{t} K=K \hat{A}_{t}^{(2)}-\hat{A}_{t}^{(1)} K
$$

Taking into account the explicit symmetric form of the Lax pair of the Bosonic Thirring model, we propose the following ansatz for the form of the defect matrix $K$ in the $\lambda$ expansion,

$$
K=K_{-1}+K_{0}+K_{1},
$$


where $K_{i}$ corresponds to an element of grade $\lambda^{i}$. Implementing the same procedure used for the Grassmaniann case, we compute the explicit form for the defect matrix $K$, and after some algebra we found the following solution:

$$
K=\left[\begin{array}{cc}
-\sqrt{\frac{m}{g}}\left[\lambda e^{-\frac{i g N_{D}}{4}}-\lambda^{-1}\left(i a^{-1}\right) e^{\frac{i g N_{D}}{4}}\right] & \lambda^{0} X \\
-\lambda^{0} X^{\dagger} & \sqrt{\frac{m}{g}}\left[\lambda e^{\frac{i g N_{D}}{4}}+\lambda^{-1}\left(i a^{-1}\right) e^{-\frac{i g N_{D}}{4}}\right]
\end{array}\right]
$$

where we have used the Bäcklund transformation (3.15)-(3.17) and the equations of motion (3.6)-(3.9).

\section{Conclusions}

In conclusion, by expressing the Bäcklund transformation for the Grassmanian Thirring model in terms of the defect number of particles we have been able to generalize this transformation to the Bosonic Thirring model. By obtaining the solutions through a dressing method which determines directly the defect number of particles we have been able to verify the Bäcklund transformation for several simple transitions, i.e. vacuum-one-soliton, onesoliton-one-soliton, one-soliton-two-solitons, and two-solitons-two-solitons.

\section{Acknowledgements}

ARA and LHY thank Fapesp, JFG and AHZ thank CNPq for partial support.

\section{A The one-soliton Solution}

Using the highest weight representation of $\hat{s} l(2)$ we obtain the one-soliton solution from the vacuum configuration, as follows ${ }^{4}$

$$
\begin{aligned}
e^{\left(\nu_{-}-\nu_{+}\right)} & =\tau_{0}, \quad e^{-2 \chi_{+}}=-\frac{\tau_{1}}{\tau_{0}}, \\
\phi_{1} & =\sqrt{\frac{m}{g}} \frac{\tau_{2}}{\tau_{1}}, \quad \phi_{2}=\sqrt{\frac{m}{g}} \frac{\tau_{4}}{\tau_{0}}, \\
\phi_{1}^{\dagger} & =\sqrt{\frac{m}{g}} \frac{\tau_{3}}{\tau_{0}}, \quad \phi_{2}^{\dagger}=\sqrt{\frac{m}{g}} \frac{\tau_{5}}{\tau_{1}},
\end{aligned}
$$

where we have introduced the tau-functions

$$
\begin{array}{lll}
\tau_{0}=\left\langle\lambda_{0}|G| \lambda_{0}\right\rangle, & \tau_{2}=\left\langle\lambda_{0}\left|\sigma_{-}^{(+1)} G\right| \lambda_{0}\right\rangle, & \tau_{4}=\left\langle\lambda_{1}\left|G \sigma_{-}^{(0)}\right| \lambda_{1}\right\rangle, \\
\tau_{1}=\left\langle\lambda_{1}|G| \lambda_{1}\right\rangle, & \tau_{3}=\left\langle\lambda_{1}\left|\sigma_{+}^{(0)} G\right| \lambda_{1}\right\rangle, & \tau_{5}=\left\langle\lambda_{0}\left|G \sigma_{+}^{(-1)}\right| \lambda_{0}\right\rangle,
\end{array}
$$

\footnotetext{
${ }^{4}$ For this solution $c$ is required to be $c=1$.
} 
and where $G=T_{0} \rho T_{0}^{-1}$ and $\rho=e^{V}$. Firstly, we can noticed that there are two possible solutions corresponding to the choice of $V=\mu_{1} V_{+}\left(\gamma_{1}\right)$, given by

$$
\nu_{+}=\nu_{-}, \quad \chi_{+}=\frac{i \pi}{2}, \quad \phi_{1}=\sqrt{\frac{m}{g}} \mu_{1} \gamma e^{-\Gamma_{1}}, \quad \phi_{2}=\sqrt{\frac{m}{g}} \mu_{1} e^{-\Gamma_{1}}, \quad \phi_{1}^{\dagger}=\phi_{2}^{\dagger}=0,
$$

and by choosing $V=\mu_{2} V_{-}\left(\gamma_{2}\right)$, we obtain

$$
\nu_{+}=\nu_{-}, \quad \chi_{+}=\frac{i \pi}{2}, \quad \phi_{1}=\phi_{2}=0, \quad \phi_{1}^{\dagger}=\sqrt{\frac{m}{g}} \mu_{2} e^{\Gamma_{2}}, \quad \phi_{2}^{\dagger}=\sqrt{\frac{m}{g}} \frac{\mu_{2}}{\gamma} e^{\Gamma_{2}} .
$$

In our case, these solutions are not interesting because of the inconsistency with the interpretation of the dagger fields $\phi^{\dagger}$ 's as the corresponding complex conjugate of the fields $\phi$ 's. Then, we construct the one-soliton solution of the system using the fact that $V_{+}(\gamma)$ and $V_{-}(-\gamma)$ have the same eigenvalue. In fact, by choosing $\rho=e^{\mu_{1} V_{+}\left(\gamma_{1}\right)} e^{\mu_{2} V_{-}\left(\gamma_{2}\right)}$ and computing the matrix elements we get the following solution,

$$
\begin{gathered}
\tau_{0}=1+\mu_{1} \mu_{2}\left[\frac{\gamma_{1} \gamma_{2}}{\left(\gamma_{1}-\gamma_{2}\right)^{2}}\right] e^{-\Gamma_{1}+\Gamma_{2}}, \quad \tau_{1}=1+\mu_{1} \mu_{2}\left[\frac{\gamma_{1}}{\gamma_{1}-\gamma_{2}}\right]^{2} e^{-\Gamma_{1}+\Gamma_{2}} \\
\tau_{0}^{(-)}=\mu_{1} \gamma_{1} e^{-\Gamma_{1}}, \quad \tau_{1}^{(-)}=\mu_{1} e^{-\Gamma_{1}}, \quad \tau_{1}^{(+)}=\mu_{2} e^{\Gamma_{2}}, \quad \tau_{0}^{(+)}=\frac{\mu_{2}}{\gamma_{2}} e^{\Gamma_{2}}
\end{gathered}
$$

where $\Gamma_{k}=i m\left(\xi \gamma_{k}+\gamma_{k}^{-1} \eta\right)$. Then, we are interested in the case where $\phi_{k}^{\dagger}$ corresponds to the complex conjugate of $\phi_{k}$, i.e., in the limit $\gamma_{2} \rightarrow-\gamma_{1}$, which provides a suitable one-soliton solution for the Bosonic Thirring model. The result is

$$
\begin{array}{rlrl}
e^{\left(\nu_{-}-\nu_{+}\right)} & =1-\Omega e^{-2 \Gamma_{1}}, & & e^{-2 \chi_{+}}=-\left[\frac{1+\Omega e^{-2 \Gamma_{1}}}{1-\Omega e^{-2 \Gamma_{1}}}\right], \\
\phi_{1} & =\sqrt{\frac{m}{g}}\left[\frac{\mu_{1} \gamma_{1} e^{-\Gamma_{1}}}{1+\Omega e^{-2 \Gamma_{1}}}\right], & \phi_{2}=\sqrt{\frac{m}{g}}\left[\frac{\mu_{1} e^{-\Gamma_{1}}}{1-\Omega e^{-2 \Gamma_{1}}}\right], \\
\phi_{1}^{\dagger}=\sqrt{\frac{m}{g}}\left[\frac{\mu_{2} e^{-\Gamma_{1}}}{1-\Omega e^{-2 \Gamma_{1}}}\right], & \phi_{2}^{\dagger}=-\sqrt{\frac{m}{g}} \frac{\mu_{2}}{\gamma_{1}}\left[\frac{e^{-\Gamma_{1}}}{1+\Omega e^{-2 \Gamma_{1}}}\right],
\end{array}
$$

where we have introduced the parameter $\Omega=\frac{\mu_{1} \mu_{2}}{4}$. Considering $m$ and $g$ to be real, and $\gamma_{1}$ purely imaginary, from (A.8-A.10) one gets that the parameters $\mu_{+}$and $\mu_{-}$must satisfy the following relation,

$$
\mu_{2}=-\gamma_{1} \mu_{1}^{*}
$$

We can also notice that for an appropriated choice of the parameters, it is possible to show the equivalence with the one-soliton solution found by Orfanidis[21].

\section{B The two-soliton Solution}

Now let us show that the two-soliton solution can be also calculated from the vacuum solution (3.23) using the dressing transformation. We will do it using only the algebraic properties 
of the affine Lie algebra $\hat{s} l(2)$. According to the approach above, there is an element $\rho$ in the group satisfying (3.34). Consider the constant group element as

$$
\rho=e^{\mu_{1} V_{+}\left(\gamma_{1}\right)} e^{\mu_{2} V_{-}\left(\gamma_{2}\right)} e^{\mu_{3} V_{+}\left(\gamma_{3}\right)} e^{\mu_{4} V_{-}\left(\gamma_{4}\right)} .
$$

The explicit form for the solution is calculated by computing the following matrix elements,

$$
\begin{aligned}
\left\langle\lambda_{0}\left|V_{+}\left(\gamma_{1}\right) V_{-}\left(\gamma_{2}\right)\right| \lambda_{0}\right\rangle & =\left\langle\lambda_{0}\left|V_{-}\left(\gamma_{2}\right) V_{+}\left(\gamma_{1}\right)\right| \lambda_{0}\right\rangle=\frac{\gamma_{1} \gamma_{2}}{\left(\gamma_{1}-\gamma_{2}\right)^{2}} \\
\left\langle\lambda_{1}\left|V_{+}\left(\gamma_{1}\right) V_{-}\left(\gamma_{2}\right)\right| \lambda_{1}\right\rangle & =\left\langle\lambda_{1}\left|V_{-}\left(\gamma_{2}\right) V_{+}\left(\gamma_{1}\right)\right| \lambda_{1}\right\rangle=\frac{\gamma_{1}^{2}}{\left(\gamma_{1}-\gamma_{2}\right)^{2}}
\end{aligned}
$$

In addition, one has

$$
\begin{array}{ll}
\left\langle\lambda_{0}\left|\sigma_{-}^{(+1)} V_{+}\left(\gamma_{1}\right)\right| \lambda_{0}\right\rangle=\gamma_{1}, & \left\langle\lambda_{1}\left|\sigma_{+}^{(0)} V_{-}\left(\gamma_{1}\right)\right| \lambda_{1}\right\rangle=1, \\
\left\langle\lambda_{0}\left|V_{-}\left(\gamma_{2}\right) \sigma_{+}^{(-1)}\right| \lambda_{0}\right\rangle=\frac{1}{\gamma_{2}}, & \left\langle\lambda_{1}\left|V_{+}\left(\gamma_{1}\right) \sigma_{-}^{(0)}\right| \lambda_{0}\right\rangle=1
\end{array}
$$

and

$$
\begin{aligned}
& \left\langle\lambda_{0}\left|V_{+}\left(\gamma_{1}\right) V_{-}\left(\gamma_{2}\right) V_{+}\left(\gamma_{3}\right) V_{-}\left(\gamma_{4}\right)\right| \lambda_{0}\right\rangle=\left[\frac{\gamma_{1} \gamma_{2} \gamma_{3} \gamma_{4}\left(\gamma_{1}-\gamma_{3}\right)^{2}\left(\gamma_{2}-\gamma_{4}\right)^{2}}{\left(\gamma_{1}-\gamma_{2}\right)^{2}\left(\gamma_{3}-\gamma_{4}\right)^{2}\left(\gamma_{1}-\gamma_{4}\right)^{2}\left(\gamma_{2}-\gamma_{3}\right)^{2}}\right] \\
& \left\langle\lambda_{1}\left|V_{+}\left(\gamma_{1}\right) V_{-}\left(\gamma_{2}\right) V_{+}\left(\gamma_{3}\right) V_{-}\left(\gamma_{4}\right)\right| \lambda_{1}\right\rangle=\left[\frac{\gamma_{1}^{2} \gamma_{3}^{2}\left(\gamma_{1}-\gamma_{3}\right)^{2}\left(\gamma_{2}-\gamma_{4}\right)^{2}}{\left(\gamma_{1}-\gamma_{2}\right)^{2}\left(\gamma_{3}-\gamma_{4}\right)^{2}\left(\gamma_{2}-\gamma_{3}\right)^{2}\left(\gamma_{1}-\gamma_{4}\right)^{2}}\right] \\
& \left\langle\lambda_{0}\left|\sigma_{-}^{(+1)} V_{+}\left(\gamma_{1}\right) V_{-}\left(\gamma_{2}\right) V_{+}\left(\gamma_{3}\right)\right| \lambda_{0}\right\rangle=\left[\frac{\gamma_{1} \gamma_{2} \gamma_{3}\left(\gamma_{1}-\gamma_{3}\right)^{2}}{\left(\gamma_{1}-\gamma_{2}\right)^{2}\left(\gamma_{2}-\gamma_{3}\right)^{2}}\right] \text {, } \\
& \left\langle\lambda_{0}\left|\sigma_{-}^{(+1)} V_{+}\left(\gamma_{1}\right) V_{+}\left(\gamma_{3}\right) V_{-}\left(\gamma_{4}\right)\right| \lambda_{0}\right\rangle=\left[\frac{\gamma_{1} \gamma_{3} \gamma_{4}\left(\gamma_{1}-\gamma_{3}\right)^{2}}{\left(\gamma_{3}-\gamma_{4}\right)^{2}\left(\gamma_{1}-\gamma_{4}\right)^{2}}\right] \text {, } \\
& \left\langle\lambda_{1}\left|\sigma_{+}^{(0)} V_{-}\left(\gamma_{2}\right) V_{+}\left(\gamma_{3}\right) V_{-}\left(\gamma_{4}\right)\right| \lambda_{1}\right\rangle=\left[\frac{\gamma_{3}^{2}\left(\gamma_{2}-\gamma_{4}\right)^{2}}{\left(\gamma_{2}-\gamma_{3}\right)^{2}\left(\gamma_{3}-\gamma_{4}\right)^{2}}\right] \text {, } \\
& \left\langle\lambda_{1}\left|\sigma_{+}^{(0)} V_{+}\left(\gamma_{1}\right) V_{-}\left(\gamma_{2}\right) V_{-}\left(\gamma_{4}\right)\right| \lambda_{0}\right\rangle=\left[\frac{\gamma_{1}^{2}\left(\gamma_{2}-\gamma_{4}\right)^{2}}{\left(\gamma_{1}-\gamma_{2}\right)^{2}\left(\gamma_{1}-\gamma_{4}\right)^{2}}\right] \text {, } \\
& \left\langle\lambda_{1}\left|V_{+}\left(\gamma_{1}\right) V_{+}\left(\gamma_{3}\right) V_{-}\left(\gamma_{4}\right) \sigma_{-}^{(0)}\right| \lambda_{1}\right\rangle=\frac{\gamma_{4}^{2}\left(\gamma_{1}-\gamma_{3}\right)^{2}}{\left(\gamma_{1}-\gamma_{4}\right)^{2}\left(\gamma_{3}-\gamma_{4}\right)^{2}} \text {, } \\
& \left\langle\lambda_{1}\left|V_{+}\left(\gamma_{1}\right) V_{-}\left(\gamma_{2}\right) V_{+}\left(\gamma_{3}\right) \sigma_{-}^{(0)}\right| \lambda_{1}\right\rangle=\frac{\gamma_{2}^{2}\left(\gamma_{1}-\gamma_{3}\right)^{2}}{\left(\gamma_{1}-\gamma_{2}\right)^{2}\left(\gamma_{2}-\gamma_{3}\right)^{2}}, \\
& \left\langle\lambda_{0}\left|V_{-}\left(\gamma_{2}\right) V_{+}\left(\gamma_{3}\right) V_{-}\left(\gamma_{4}\right) \sigma_{+}^{(-1)}\right| \lambda_{0}\right\rangle=\frac{\gamma_{3}^{3}\left(\gamma_{2}-\gamma_{4}\right)^{2}}{\gamma_{2} \gamma_{4}\left(\gamma_{2}-\gamma_{3}\right)^{2}\left(\gamma_{3}-\gamma_{4}\right)^{2}} \text {, } \\
& \left\langle\lambda_{0}\left|V_{+}\left(\gamma_{1}\right) V_{-}\left(\gamma_{2}\right) V_{-}\left(\gamma_{4}\right) \sigma_{+}^{(-1)}\right| \lambda_{0}\right\rangle=\frac{\gamma_{1}^{3}\left(\gamma_{2}-\gamma_{4}\right)^{2}}{\gamma_{2} \gamma_{4}\left(\gamma_{1}-\gamma_{2}\right)^{2}\left(\gamma_{1}-\gamma_{4}\right)^{2}},
\end{aligned}
$$


So, we obtain the following results,

$$
\begin{aligned}
& \tau_{0}=1+\mu_{1} \mu_{2} e^{-\Gamma_{1}+\Gamma_{2}}\left[\frac{\gamma_{1} \gamma_{2}}{\left(\gamma_{1}-\gamma_{2}\right)^{2}}\right]+\mu_{1} \mu_{4} e^{-\Gamma_{1}+\Gamma_{4}}\left[\frac{\gamma_{1} \gamma_{4}}{\left(\gamma_{1}-\gamma_{4}\right)^{2}}\right] \\
& +\mu_{2} \mu_{3} e^{\Gamma_{2}-\Gamma_{3}}\left[\frac{\gamma_{2} \gamma_{3}}{\left(\gamma_{2}-\gamma_{3}\right)^{2}}\right]+\mu_{3} \mu_{4} e^{-\Gamma_{3}+\Gamma_{4}}\left[\frac{\gamma_{3} \gamma_{4}}{\left(\gamma_{3}-\gamma_{4}\right)^{2}}\right] \\
& +\mu_{1} \mu_{2} \mu_{3} \mu_{4} e^{-\Gamma_{1}+\Gamma_{2}-\Gamma_{3}+\Gamma_{4}}\left[\frac{\gamma_{1} \gamma_{2} \gamma_{3} \gamma_{4}\left(\gamma_{1}-\gamma_{3}\right)^{2}\left(\gamma_{2}-\gamma_{4}\right)^{2}}{\left(\gamma_{1}-\gamma_{2}\right)^{2}\left(\gamma_{3}-\gamma_{4}\right)^{2}\left(\gamma_{1}-\gamma_{4}\right)^{2}\left(\gamma_{2}-\gamma_{3}\right)^{2}}\right] \text {, } \\
& \tau_{1}=1+\mu_{1} \mu_{2} e^{-\Gamma_{1}+\Gamma_{2}}\left[\frac{\gamma_{1}^{2}}{\left(\gamma_{1}-\gamma_{2}\right)^{2}}\right]+\mu_{1} \mu_{4} e^{-\Gamma_{1}+\Gamma_{4}}\left[\frac{\gamma_{1}^{2}}{\left(\gamma_{1}-\gamma_{4}\right)^{2}}\right] \\
& +\mu_{2} \mu_{3} e^{\Gamma_{2}-\Gamma_{3}}\left[\frac{\gamma_{3}^{2}}{\left(\gamma_{2}-\gamma_{3}\right)^{2}}\right]+\mu_{3} \mu_{4} e^{-\Gamma_{3}+\Gamma_{4}}\left[\frac{\gamma_{3}^{2}}{\left(\gamma_{3}-\gamma_{4}\right)^{2}}\right] \\
& +\mu_{1} \mu_{2} \mu_{3} \mu_{4} e^{-\Gamma_{1}+\Gamma_{2}-\Gamma_{3}+\Gamma_{4}}\left[\frac{\gamma_{1}^{2} \gamma_{3}^{2}\left(\gamma_{1}-\gamma_{3}\right)^{2}\left(\gamma_{2}-\gamma_{4}\right)^{2}}{\left(\gamma_{1}-\gamma_{2}\right)^{2}\left(\gamma_{3}-\gamma_{4}\right)^{2}\left(\gamma_{2}-\gamma_{3}\right)^{2}\left(\gamma_{1}-\gamma_{4}\right)^{2}}\right] \\
& \tau_{2}=\mu_{1} \gamma_{1} e^{-\Gamma_{1}}+\mu_{3} \gamma_{3} e^{-\Gamma_{3}}+\mu_{1} \mu_{2} \mu_{3} e^{-\Gamma_{1}+\Gamma_{2}-\Gamma_{3}}\left[\frac{\gamma_{1} \gamma_{2} \gamma_{3}\left(\gamma_{1}-\gamma_{3}\right)^{2}}{\left(\gamma_{1}-\gamma_{2}\right)^{2}\left(\gamma_{2}-\gamma_{3}\right)^{2}}\right] \\
& +\mu_{1} \mu_{3} \mu_{4} e^{-\Gamma_{1}-\Gamma_{3}+\Gamma_{4}}\left[\frac{\gamma_{1} \gamma_{3} \gamma_{4}\left(\gamma_{1}-\gamma_{3}\right)^{2}}{\left(\gamma_{3}-\gamma_{4}\right)^{2}\left(\gamma_{1}-\gamma_{4}\right)^{2}}\right] \\
& \tau_{3}=\mu_{2} e^{\Gamma_{2}}+\mu_{4} e^{\Gamma_{4}}+\mu_{2} \mu_{3} \mu_{4} e^{\Gamma_{2}-\Gamma_{3}+\Gamma_{4}}\left[\frac{\gamma_{3}^{2}\left(\gamma_{2}-\gamma_{4}\right)^{2}}{\left(\gamma_{2}-\gamma_{3}\right)^{2}\left(\gamma_{3}-\gamma_{4}\right)^{2}}\right] \\
& +\mu_{1} \mu_{2} \mu_{4} e^{-\Gamma_{1}+\Gamma_{2}+\Gamma_{4}}\left[\frac{\gamma_{1}^{2}\left(\gamma_{2}-\gamma_{4}\right)^{2}}{\left(\gamma_{1}-\gamma_{2}\right)^{2}\left(\gamma_{1}-\gamma_{4}\right)^{2}}\right] \\
& \tau_{4}=\mu_{1} e^{-\Gamma_{1}}+\mu_{3} e^{-\Gamma_{3}}+\mu_{1} \mu_{2} \mu_{3} e^{-\Gamma_{1}+\Gamma_{2}-\Gamma_{3}}\left[\frac{\gamma_{2}^{2}\left(\gamma_{1}-\gamma_{3}\right)^{2}}{\left(\gamma_{1}-\gamma_{2}\right)^{2}\left(\gamma_{2}-\gamma_{3}\right)^{2}}\right] \\
& +\mu_{1} \mu_{3} \mu_{4} e^{-\Gamma_{1}-\Gamma_{3}+\Gamma_{4}}\left[\frac{\gamma_{4}^{2}\left(\gamma_{1}-\gamma_{3}\right)^{2}}{\left(\gamma_{1}-\gamma_{4}\right)^{2}\left(\gamma_{3}-\gamma_{4}\right)^{2}}\right] \\
& \tau_{5}=\frac{\mu_{2}}{\gamma_{2}} e^{\Gamma_{2}}+\frac{\mu_{4}}{\gamma_{4}} e^{\Gamma_{4}}+\mu_{2} \mu_{3} \mu_{4} e^{\Gamma_{2}-\Gamma_{3}+\Gamma_{4}}\left[\frac{\gamma_{3}^{3}\left(\gamma_{2}-\gamma_{4}\right)^{2}}{\gamma_{2} \gamma_{4}\left(\gamma_{2}-\gamma_{3}\right)^{2}\left(\gamma_{3}-\gamma_{4}\right)^{2}}\right] \\
& +\mu_{1} \mu_{2} \mu_{4} e^{-\Gamma_{1}+\Gamma_{2}+\Gamma_{4}}\left[\frac{\gamma_{1}^{3}\left(\gamma_{2}-\gamma_{4}\right)^{2}}{\gamma_{2} \gamma_{4}\left(\gamma_{1}-\gamma_{2}\right)^{2}\left(\gamma_{1}-\gamma_{4}\right)^{2}}\right]
\end{aligned}
$$

We can check that these tau-functions satisfy the equations (3.6)-(3.9) and (3.31) for any values of the parameters $\mu_{k}$ and $\gamma_{k}$, with $k=1, \ldots, 4$. Now, taking the limit $\gamma_{2} \rightarrow-\gamma_{1}$ and $\gamma_{4} \rightarrow-\gamma_{3}$, we get the two-soliton solution for the Bosonic Thirring model. The tau-functions become,

$$
\tau_{0}=1-\frac{\mu_{1} \mu_{2}}{4} e^{-2 \Gamma_{1}}-\frac{\mu_{3} \mu_{4}}{4} e^{-2 \Gamma_{3}}-\left(\mu_{1} \mu_{4}+\mu_{2} \mu_{3}\right) e^{-\left(\Gamma_{1}+\Gamma_{3}\right)}\left[\frac{\gamma_{1} \gamma_{3}}{\left(\gamma_{1}+\gamma_{3}\right)^{2}}\right]
$$




$$
\begin{aligned}
& +\frac{1}{16}\left(\mu_{1} \mu_{2} \mu_{3} \mu_{4}\right) e^{-2\left(\Gamma_{1}+\Gamma_{3}\right)}\left[\frac{\gamma_{1}-\gamma_{3}}{\gamma_{1}+\gamma_{3}}\right]^{4} \\
& \tau_{1}=1+\frac{\mu_{1} \mu_{2}}{4} e^{-2 \Gamma_{1}}+\frac{\mu_{3} \mu_{4}}{4} e^{-2 \Gamma_{3}}+\left(\gamma_{1}^{2} \mu_{1} \mu_{4}+\gamma_{3}^{2} \mu_{2} \mu_{3}\right) e^{-\left(\Gamma_{1}+\Gamma_{3}\right)}\left[\frac{1}{\left(\gamma_{1}+\gamma_{3}\right)^{2}}\right] \\
& +\frac{1}{16}\left(\mu_{1} \mu_{2} \mu_{3} \mu_{4}\right) e^{-2\left(\Gamma_{1}+\Gamma_{3}\right)}\left[\frac{\gamma_{1}-\gamma_{3}}{\gamma_{1}+\gamma_{3}}\right]^{4} \\
& \tau_{2}=\mu_{1} \gamma_{1} e^{-\Gamma_{1}}+\mu_{3} \gamma_{3} e^{-\Gamma_{3}}-\frac{1}{4} \mu_{1} \mu_{2} \mu_{3} e^{-\left(2 \Gamma_{1}+\Gamma_{3}\right)}\left[\frac{\gamma_{3}\left(\gamma_{1}-\gamma_{3}\right)^{2}}{\left(\gamma_{1}+\gamma_{3}\right)^{2}}\right] \\
& -\frac{1}{4} \mu_{1} \mu_{3} \mu_{4} e^{-\left(\Gamma_{1}+2 \Gamma_{3}\right)}\left[\frac{\gamma_{1}\left(\gamma_{1}-\gamma_{3}\right)^{2}}{\left(\gamma_{1}+\gamma_{3}\right)^{2}}\right] \\
& \tau_{3}=\mu_{2} e^{-\Gamma_{1}}+\mu_{4} e^{-\Gamma_{3}}+\frac{1}{4} \mu_{2} \mu_{3} \mu_{4} e^{-\left(\Gamma_{1}+2 \Gamma_{3}\right)}\left[\frac{\gamma_{1}-\gamma_{3}}{\gamma_{1}+\gamma_{3}}\right]^{2} \\
& +\frac{1}{4} \mu_{1} \mu_{2} \mu_{4} e^{-\left(2 \Gamma_{1}+\Gamma_{3}\right)}\left[\frac{\gamma_{1}-\gamma_{3}}{\gamma_{1}+\gamma_{3}}\right]^{2}, \\
& \tau_{4}=\mu_{1} e^{-\Gamma_{1}}+\mu_{3} e^{-\Gamma_{3}}+\frac{1}{4} \mu_{1} \mu_{2} \mu_{3} e^{-\left(2 \Gamma_{1}+\Gamma_{3}\right)}\left[\frac{\gamma_{1}-\gamma_{3}}{\gamma_{1}+\gamma_{3}}\right]^{2} \\
& +\frac{1}{4} \mu_{1} \mu_{3} \mu_{4} e^{-\left(\Gamma_{1}+2 \Gamma_{3}\right)}\left[\frac{\gamma_{1}-\gamma_{3}}{\gamma_{1}+\gamma_{3}}\right]^{2}, \\
& \tau_{5}=-\frac{\mu_{2}}{\gamma_{1}} e^{-\Gamma_{1}}-\frac{\mu_{4}}{\gamma_{3}} e^{-\Gamma_{3}}+\frac{1}{4} \mu_{2} \mu_{3} \mu_{4} e^{-\left(\Gamma_{1}+2 \Gamma_{3}\right)}\left[\frac{\left(\gamma_{1}-\gamma_{3}\right)^{2}}{\gamma_{1}\left(\gamma_{1}+\gamma_{3}\right)^{2}}\right] \\
& +\frac{1}{4} \mu_{1} \mu_{2} \mu_{4} e^{-\left(2 \Gamma_{1}+\Gamma_{3}\right)}\left[\frac{\left(\gamma_{1}-\gamma_{3}\right)^{2}}{\gamma_{3}\left(\gamma_{1}+\gamma_{3}\right)^{2}}\right] \text {. }
\end{aligned}
$$

Considering that $m$ and $g$ must be real constants, there are two possibilities in order to $\phi_{k}^{\dagger}$ does corresponds to the complex conjugate of $\phi_{k}$. First one corresponds to the case of $\gamma_{1}$ and $\gamma_{3}$ to be purely imaginary numbers, and the parameters $\mu_{k}$ satisfyng the following conditions,

$$
\mu_{2}=-\gamma_{1} \mu_{1}^{*}, \quad \mu_{4}=-\gamma_{3} \mu_{3}^{*} .
$$

The second possibility corresponds to the situation when $\gamma_{3}^{*}=-\gamma_{1}$, and as consequence $\Gamma_{3}^{*}=\Gamma_{1}$. In this case, we need that the parameters $\mu_{k}$ satisfy the following conditions,

$$
\mu_{4}^{*}=\gamma_{1} \mu_{1}, \quad \mu_{2}^{*}=\gamma_{3} \mu_{3} .
$$




\section{The $s l(2,1)$ affine Lie algebra notations}

Consider the $\hat{s} l(2,1)$ super Lie algebra with its generators given by

$$
\begin{aligned}
h_{1} & =\alpha_{1} \cdot H=\left(\begin{array}{rrr}
1 & 0 & 0 \\
0 & -1 & 0 \\
0 & 0 & 0
\end{array}\right), \quad h_{2}=\alpha_{2} \cdot H=\left(\begin{array}{lll}
0 & 0 & 0 \\
0 & 1 & 0 \\
0 & 0 & 1
\end{array}\right), \\
E_{\alpha_{1}} & =\left(\begin{array}{lll}
0 & 1 & 0 \\
0 & 0 & 0 \\
0 & 0 & 0
\end{array}\right), E_{-\alpha_{1}}=\left(\begin{array}{lll}
0 & 0 & 0 \\
1 & 0 & 0 \\
0 & 0 & 0
\end{array}\right), \quad E_{\alpha_{2}}=\left(\begin{array}{lll}
0 & 0 & 0 \\
0 & 0 & 1 \\
0 & 0 & 0
\end{array}\right), \\
E_{-\alpha_{2}} & =\left(\begin{array}{lll}
0 & 0 & 0 \\
0 & 0 & 0 \\
0 & 1 & 0
\end{array}\right), E_{\alpha_{1}+\alpha_{2}}=\left(\begin{array}{lll}
0 & 0 & 1 \\
0 & 0 & 0 \\
0 & 0 & 0
\end{array}\right), \quad E_{-\left(\alpha_{1}+\alpha_{2}\right)}=\left(\begin{array}{lll}
0 & 0 & 0 \\
0 & 0 & 0 \\
1 & 0 & 0
\end{array}\right),
\end{aligned}
$$

where $\alpha_{1}$ is a bosonic root and $\alpha_{2}, \alpha_{1}+\alpha_{2}$ are the fermionic roots. The affine $\hat{s} l(2,1)$ algebra is decomposed according to the grading operator

$$
Q=2 d+\frac{1}{2} h_{1}
$$

where $d$ is the derivation operator satisfying $\left[d, T_{a}^{(n)}\right]=n T_{a}^{(n)}$. Here $T_{a}^{(n)}$ denotes both $H_{i}^{(n)}$ and $E_{\alpha}^{(n)}$. The hierarchy is further specified by the constant grade one element $E=E^{(1)}$, as follows

$$
E^{(2 n+1)}=h_{1}^{(n+1 / 2)}+2 h_{2}^{(n+1 / 2)}=K_{2}^{(2 n+1)},
$$

here $\mu_{i}$ denotes the $i$-th fundamental weight. The grading operator $Q$ together with the judicious choice of $E$ decomposes the affine super Kac-Moody algebra $\hat{\mathcal{G}}=\hat{s} l(2,1)$ into $\hat{\mathcal{G}}=\mathcal{K} \oplus \mathcal{M}$, where the Kernel $\mathcal{K}=\{x \in \hat{\mathcal{G}} \mid[x, E]=0\}$ of $\mathrm{E}$, and its complement $\mathcal{M}$ are given by

$$
\begin{aligned}
\mathcal{K} & =\left\{K_{1}^{(2 n+1)}, K_{2}^{(2 n+1)}, M_{1}^{(2 n+1)}, M_{2}^{(2 n)}\right\} \\
\mathcal{M} & =\left\{F_{1}^{(2 n+3 / 2)}, F_{2}^{(2 n+1 / 2)}, G_{1}^{(2 n+1 / 2)}, G_{2}^{(2 n+3 / 2)}\right\}
\end{aligned}
$$

where the bosonic generators are

$$
\begin{array}{lll}
M_{1}^{(2 n+1)} & =-\left(E_{\alpha_{1}}^{(n)}-E_{-\alpha_{1}}^{(n+1)}\right), & M_{2}^{(2 n)}=h_{1}^{(n)}, \\
K_{1}^{(2 n+1)}=-\left(E_{\alpha_{1}}^{(n)}+E_{-\alpha_{1}}^{(n+1)}\right), & K_{2}^{(2 n+1)}=\mu_{2} \cdot H^{(n+1 / 2)},
\end{array}
$$

and the fermionic generators are

$$
\begin{aligned}
& F_{1}^{(2 n+3 / 2)}=\left(E_{\alpha_{1}+\alpha_{2}}^{(n+1 / 2)}-E_{\alpha_{2}}^{(n+1)}\right)+\left(E_{-\left(\alpha_{1}+\alpha_{2}\right)}^{(n+1)}-E_{-\alpha_{2}}^{(n+1 / 2)}\right), \\
& F_{2}^{(2 n+1 / 2)}=-\left(E_{\alpha_{1}+\alpha_{2}}^{(n)}-E_{\alpha_{2}}^{(n+1 / 2)}\right)+\left(E_{-\left(\alpha_{1}+\alpha_{2}\right)}^{(n+1 / 2)}-E_{-\alpha_{2}}^{(n)}\right), \\
& G_{1}^{(2 n+1 / 2)}=\left(E_{\alpha_{1}+\alpha_{2}}^{(n)}+E_{\alpha_{2}}^{(n+1 / 2)}\right)+\left(E_{-\left(\alpha_{1}+\alpha_{2}\right)}^{(n+1 / 2)}+E_{-\alpha_{2}}^{(n)}\right), \\
& G_{2}^{(2 n+3 / 2)}=-\left(E_{\alpha_{1}+\alpha_{2}}^{(n+1 / 2)}+E_{\alpha_{2}}^{(n+1)}\right)+\left(E_{-\left(\alpha_{1}+\alpha_{2}\right)}^{(n+1)}+E_{-\alpha_{2}}^{(n+1 / 2)}\right)
\end{aligned}
$$




\section{References}

[1] E. Corrigan "Integrable Models with boundaries", Math-ph 0411043

[2] P. Bowcock, E. Corrigan, C. Zambon, Int. J. Mod. Phys., A19S2, (2004), 82, and [hep-th/0305022]; JHEP, 0401, (2004), 056, and [hep-th/0401020].

[3] E. Corrigan and C. Zambon, J. Phys., A37, (2004), L471, and [hepth/0407199];Nonlinearity, 19, (2006), 1447, and [nli.SI/0512038].

[4] P. Bowcock, E. Corrigan, P.E. Dorey, RH Rietdijk, Nucl.Phys., B445, (1995), 469, and [hep-th/9501098].

[5] P. Bowcock, E. Corrigan, and C. Zambon, Int.J.Mod.Phys., A19S2, (2004) 82, and [hep-th/0305022].

[6] Z. Bajnok and Z. Simon, Nucl. Phys. B802 (2008) 307

[7] F. Nemes, Semiclassical Analysis of Defect sine-Gordon Theory, [hep-th/0909.3268].

[8] V. Caudrelier, IJGMMP, vol.5, No. 7, (2008) 1085-1108.

[9] J. F. Gomes, L. H. Ymai, and A. H. Zimerman, J. Phys. A : Math. Gen., 39, (2006) 7471, and [hep-th/0601014], see also J. F. Gomes, L. H. Ymai, A. H. Zimerman, "Classical Integrable $\mathrm{N}=1$ and $N=2$ Super Sinh-Gordon Models with Jump Defects", J.Phys.Conf.Ser.128:012004,2008, [arXiv:0708.2407].

[10] J. F. Gomes, L. H. Ymai, and A. H. Zimerman, JHEP 0803:001,2008 , [arXiv:0710.1391] (hep-th).

[11] A.R. Aguirre, J.F. Gomes, L.H. Ymai, e A. H. Zimerman, Proceedings of Science, PoS(ISFTG)031 (2009), [nlin.SI : 0910.2888v2].

[12] A. Izergin and J. Stehr, DESY 76/60, Nov 1976. 8pp.

[13] A.Z. Izergin and P.P. Kulish, Letters in Math. Phys. 2 (1978) 297

[14] S.R. Coleman, Phys. Rev. D11 (1975) 2088

[15] E.A. Kuznetsov and A.V. Mihailov, Theor. Mat. Phys. 30 (1977) 193

[16] O. Babelon and D. Bernard, Int. J. Mod. Phys. A8 (1993) 507, see also L. A. Ferreira, J.L. Miramontes and J. Sanchez Guillen, J. Math. Phys. 38 (1997) 882, [e-Print: hepth/9606066].

[17] S. Mandelstam, Phys. Rev. D 11, 30263030 (1975)

[18] M.J. Ablowitz, D.J. Kaup, A.C Newell, H. Segur, Stud. Appl. Math., 53, (1975), 249.

[19] P.P. Kulish and E.K. Sklyanin, "Quantum Spectral Transform Method. Recent Developments", Lecture Notes in Physics, Vol. 151 (Springer-Verlag, Berlin, 1982), p. 61. 
[20] H.H. Chen, "General Derivation of Bäcklund Transformations from Inverse Scattering Method", Phys. Rev. Lett., 33, (1974), 925.

[21] S.J. Orfanidis, Phys. Rev., D14, No. 2 (1976), 472.

[22] M. Wadati and K. Sogo, J. Phys. Soc. Jpn, 52, (1983), 394-398. 